\title{
A Typical Kronlein Shot? A Rare Case of Submental-Facio-Cranial Bullet Trajectory with Brain Exenteration
}

Luv Sharma* and Tarun Dagar

Department of Forensic Medicine, Pt. B.D. Sharma Post-Graduate Institute of Medical Sciences, University of Health Sciences, Rohtak, Haryana, India

"Corresponding author: Dr. Luv Sharma, Professor, Department of Forensic Medicine, Pt. B.D. Sharma Post-Graduate Institute of Medical Sciences, University of Health Sciences, Rohtak, Haryana, India, Tel: 919416101258; E-mail: drluvksharma@yahoo.com

Rec date: Feb 22, 2015 Acc date: June 27, 2015 Pub date: July 02, 2015

Copyright: $\odot 2015$ Sharma L, et al. This is an open-access article distributed under the terms of the Creative Commons Attribution License, which permits unrestricted use, distribution, and reproduction in any medium, provided the original author and source are credited.

\begin{abstract}
The "Krönlein" shot (evisceration of the brain) is a very rare injury of the skull caused by a high-velocity bullet. This type of low-range shot wound involves is a broad opening of the skull with laceration of the dura mater. In the past, several cases of this particular injury have been reported and all led to immediate death. $R U$ Kronlein first described this phenomenon in 1889. Since then, this unique firearm wound and its mechanics have been discussed from time to time. The authors present an atypical presentation of Kronlein shot, wherein the entry wound was situated on the submandibular triangle instead of the typical head region. The bullet track was through the floor of mouth, further on to enter the cranial cavity to cause a blowout fracture defect with the brain being eviscerated out of the skull.
\end{abstract}

Keywords: Firearm injury; Exenteration; Brain

\section{Introduction}

Firearm wounds to the head invariably have typical entry and exit wounds or solely entry wounds when the projectile has not ejected the skull. Usually, distinctive features present on skin and bone are available to the autopsy surgeon to distinguish between these wounds. Ballistics is however an intriguing science. Multiple factors like the type of ammunition used, muzzle velocity and target characteristics play a role in the final outcome of a gunshot. The resultant wound(s) may show atypical features where exact demarcation of entry and exit wounds may not be possible on gross appearance. One such injury to the head is the "Kronlein Shot" which is a low-range shot wound. The energy deposited in the wound as the bullet passes through the flesh imparts a momentum to the tissues directed radially outward from the bullet's path. This energy and momentum is that a temporary cavity is formed during the bullet's passage. The consequence of cavitation in an enclosed space is predictable. It follows from the fact that a liquid can't be compressed. A forceful wave of hydraulic pressure is applied to the walls of the enclosure, in this case the bony cranium causing it to burst open. The effect is further facilitated by the fractures radiating out from the point of penetration and the extension of these fractures by the visco-elastic deformation of the skull at impact. The fractures act as lines of weakness which give way under the pressure applied by the underlying tissues and body fluids driven outward against the inner wall of the skull [1-3].

This paper reports a bilaterally symmetrical cranio-facial fracture pattern that is observed in a self-inflicted, midline gunshot entry wound placed on the submandibular (Digastric) triangle with resultant exenteration of the brain.

\section{Case Report}

Referral: The case was referred to our department for expert postmortem examination from a peripheral district hospital with the following referral note - "Head and neck totally shattered and parts of head are unidentifiable. Not possible to find cause of death in this case of alleged firearm injury."

Findings on autopsy: The shirt worn by the deceased was smudged with brain tissue and stained with blood more on the anterior aspect. The body was received in a single segment (Figure 1). The entire cranio-facial structures were missing with only infra-labial labial region including side of the facial area extending backwards uptil lower occipital area present. Consequently only lower postero-lateral and posterior tissues were present. Of the cranial bones, only lower occipital bones were identifiable which showed extensive fractures (Figure 2). The sub-mandibular region was disrupted. On approximation of this disrupted sub-mandibular and infra-labial area, the entry wound of firearm was reconstructed as an irregular wound over base of the chin in the sub-mandibular region in the midline with extensively lacerated, contused and ragged margins of size $3 \times 3 \mathrm{~cm}$ with appreciable impression of the muzzle of width $0.25 \mathrm{~cm}$ after reconstruction (Figure 3). Just to the right of this wound, the skin showed a distinct semi-lunar raised area of diameter $2.5 \mathrm{~cm}$ with a muzzle impression on the antero-lateral margin (Figure 4). This wound track was traced with the track found to be directed upwards and backwards to the shatter the mandible into multiple pieces with infiltration of blood into exposed trabeculae of fractured bone segments. There was complete disruption of the cranio-facial structures causing a 'blow- out' defect representing the exit wound. the whole re-contructed track of this wound showed cherry red discoloration in the available remnants of muscle and soft tissue. The available brain substance was severely lacerated and disrupted with a major part blown off and missing. From this disrupted mass of brain tissue, three deformed metallic pellets were recovered which were sent for ballistic analysis. Several small pieces of soft tissue and bone were received separately. These represented parts of the missing facial area and were identified as shattered cranial and facial bone fragments, teeth and nasal skin. All the bone fragments showed infiltration of blood. 
Citation: Sharma L, Dagar T (2015) A Typical Kronlein Shot? A Rare Case of Submental-Facio-Cranial Bullet Trajectory with Brain Exenteration. J Forensic Res S3: 1000S3-002. doi:10.4172/2157-7145.1000S3-002

Page 2 of 3

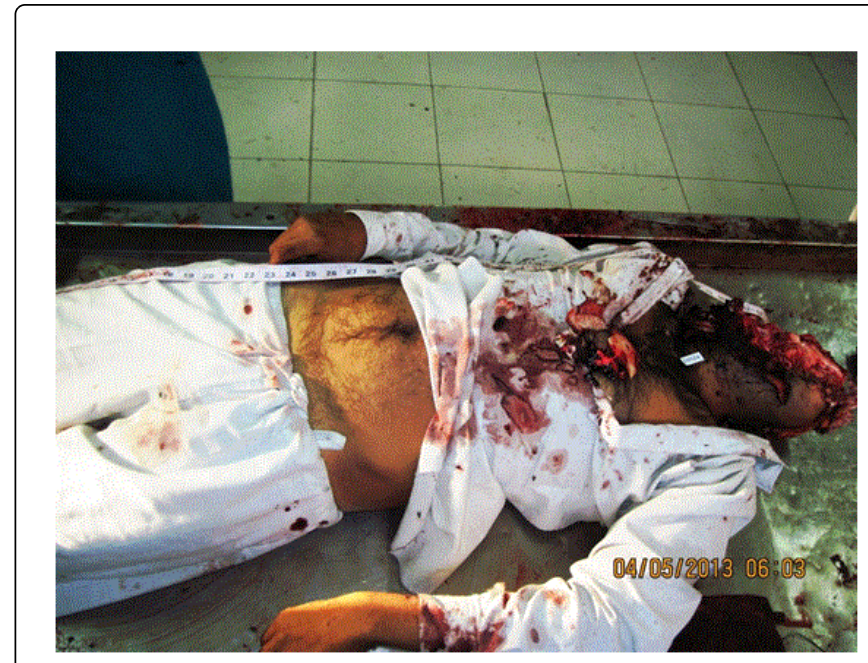

Figure 1: The dead body as received in the mortuary

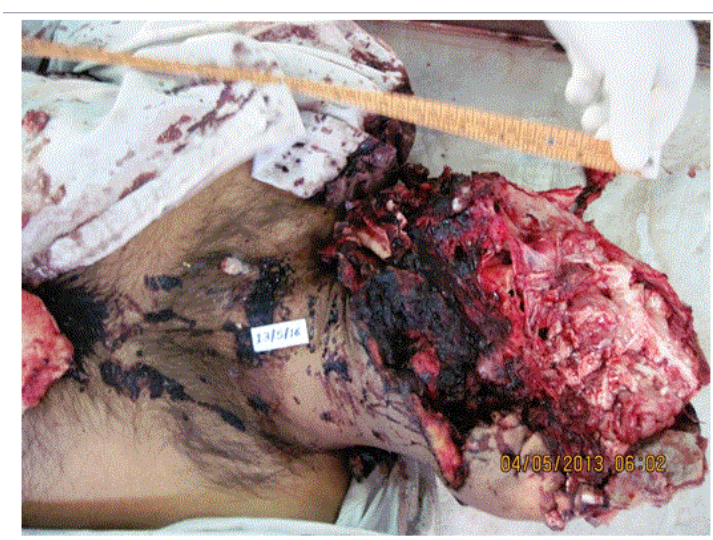

Figure 2: The head and neck region showing blowout defect

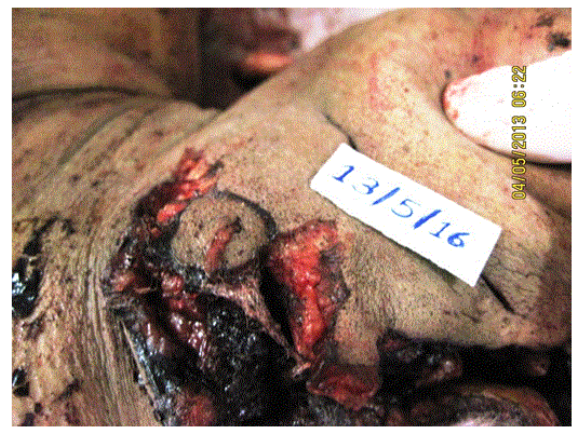

Figure 3: Reconstructed entry wound over the submandibular region. The distinct muzzle impression of the double barrel shotgun has been reconstructed by approximation of the torn skin margins.

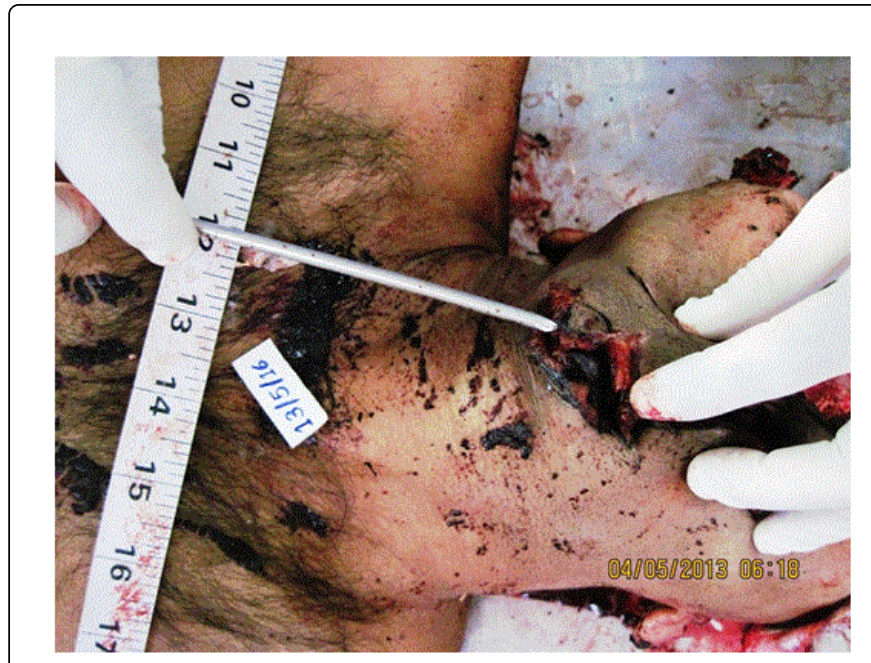

Figure 4: The track of the wound depicted after approximation of the disrupted edges.

After the ballistic report was received, we opined the cause of death as 'firearm injury and consequent cranio-facial disruption.

\section{Discussion}

Atypical gunshot wounds of entrance occur when bullets deviate from their stable nose-on trajectory before entering the body. When this occurs, the resulting wound may have an atypical D-shaped appearance. Ray-like abrasions or bruises may radiate from the corners of the wound. Unstable non-axial flight may be caused by intermediate targets, ricochets, inappropriate weapon/ ammunition combinations, poor weapon construction, or use of misaligned silencers. If a bullet is deformed before entrance the configuration of the resulting wound may be bizarre, and the wound configuration may closely resemble the configuration of the striking bullet [4].

The appearance of gunshot entrance wounds on the body depends upon many factors including, the type of firearm, the type of ammunition, the location of the wound on the body, and the circumstances of how a wound was sustained. Atypical gunshot entrance wounds are usually created when the bullet is destabilized prior to entering the body and consequently does not enter the body nose first but sideways or at an angle. The most common causes of an atypical entrance wound are bullet ricochet and interaction with an intermediate target. A case is presented in which the decedent sustained a gunshot wound with an atypical entrance. The cause of the atypical nature of the wound was determined to be increased yaw due to bullet instability caused by the condition of the firearm used, not a ricochet or intermediate target. The case emphasizes the importance of collaborative investigation between the different forensic agencies in gunshot cases [5].

Marquart et al studied 20 gunshot wounds which showed stretchmark-like tears of the facial skin away from the entrance wound localised either in the mouth, the forehead, or the submental region. The stretchmark-like tears were found in the region of the eyes and the nasolabial folds. Radial tears were seen on the lips and in the vicinity of the corners of the mouth, particularly in cases involving shots into the mouth.. They were apparently caused by the subcutaneous or intraoral 
expansion of the muzzle gases and/or the radial forces of the bullet resulting in ballooning and overextension of the facial soft tissues. The weapons used were not only rifles and shotguns, but also revolvers and pistols [6].

Cullen et al report a firearm fatality by suicide using a homemade shotgun. Autopsy revealed a contact shotgun wound to the right side of the decedent's head. The shotgun blast caused injury to the skull with evisceration of the brain [7].

Ventura et al describe a case of a suicide with a shotgun charged with 9 caliber Flobert cartridge that had been discharged in contact with the roof of the mouth causing not particularly destroying cranium-encephalic wounds [8].

Farrugia et al report a case with an atypical entrance wound as a result of a destabilized full metal jacket bullet penetration. The destabilized bullet by an impact with the dorsal hand experienced a yawing to tumbling motion in flight. The large angle of yaw induced a larger presenting profile upon impact that contributed, associated to a rapid deceleration, to a greater mechanical force on the projectile structure and a fragmentation into core and jacket. Forensic pathologists have to be aware that the metal jacket bullet could tend to break up outside or inside the body particularly after a shooting through a target. This phenomenon induces atypical entrance wounds and atypical X-ray presentation [9].

An alleged case of firearm death mimicked injury caused by an explosion, and if the case was that caused by a firearm (as alleged by the police), the main difficulty was to find the entry and exit wounds, which were obliterated due to the explosive effects. On internal examination, both cerebral hemispheres were found to be missing. However cerebellum below tentorium was intact. The injury was reconstructed by approximating the extruded margins of the scalp. Upon reconstruction, both entry and exit wounds could be seen easily. Both entry and exit wounds in the scalp were found to be in line with the corresponding defects in the skull. The case was that of a typical Kronlein shot [10].

Autopsy in another case of blowout firearm injury to the head was conducted by the author in which a person accidently blew his brains out in a fit of rage. The entry wound of firearm (after approximation of skin margins with reconstruction was present over the frontal region of scalp, just left to midline, with ragged, irregular and contused margins. Abraded collar of width $2-3 \mathrm{~cm}$ at places was present around the wound margins. The wound was directed backwards and upwards to enter the cranial cavity through the frontal bones, lacerating both cerebral hemispheres (more on left side) to cause a blow-out fracture in the vertex region of scalp (representing exit wound of this firearm) [11].

\section{References}

1. Pankratz H, Fischer H (1985) [Wound ballistics of the Krönlein shot]. Z Rechtsmed 95: 213-215.

2. Hildebrandt G, Stienen MN, Patzer J, Surbeck W (2012) Neurosurgical contributions of the Swiss surgeon, Rudolf Ulrich Krönlein--a further pioneer in Swiss neurosurgery. Acta Neurochir (Wien) 154: 1923-1933.

3. Quatrehomme G IÅŸcan MY (1999) Characteristics of gunshot wounds in the skull. J Forensic Sci 44: 568-576.

4. Donoghue ER, Kalelkar MB, Richmond JM, Teas SS (1984) Atypical gunshot wounds of entrance: an empirical study. J Forensic Sci 29: 379-388.

5. Molina DK Rulon JJ, Wallace EI (2012) The atypical entrance wound: differential diagnosis and discussion of an unusual cause. Am J Forensic Med Pathol 33: 250-252.

6. Faller-Marquardt M Pollak S (2002) Skin tears away from the entrance wound in gunshots to the head. Int J Legal Med 116: 262-266.

7. Cullen EF Luckasevic TM (2010) Suicide with a homemade shotgun: case report and review of literature. Am J Forensic Med Pathol 31: 255-257.

8. Ventura F Rocca G, Ventura A, Celesti R (2011) Suicide with "Florbert shotgun": case report. Am J Forensic Med Pathol 32: 321-323.

9. Farrugia A Raul JS, Geraut A, Tortel MC, Ludes B (2009) Destabilization and intracranial fragmentation of a full metal jacket bullet. J Forensic Leg Med 16: 400-402.

10. Sirohiwal BL, Sirohiwal D, Sharma L, Paliwal PK, Sirohiwal S (2008) A Rare Presentation of A Firearm Injury of The Head. Anil Aggrawal's Internet Journal of Forensic Medicine and Toxicology [serial online] 9: 7

11. Sharma L, Garg V, Vashisht Y (2014) Accidental Kronlein Shot. Malaysian Journal of Forensic Sciences 5: 1-5.
This article was originally published in a special issue, entitled: "Analytical Applications in Forensic Sciences", Edited by Harvey Hou, Alabama State University , USA 